\title{
High purity $x$-ray polarimetry with single-crystal diamonds
}

Hendrik Bernhardt, ${ }^{1,2, a)}$ Berit Marx-Glowna, ${ }^{1}$ Kai S. Schulze, ${ }^{1,2}$ Benjamin Grabiger, ${ }^{1,2}$ Johann Haber, ${ }^{3}$ Carsten Detlefs, ${ }^{4}$ Robert Loetzsch, ${ }^{1,2}$ Tino Kämpfer, ${ }^{1,2}$ Ralf Röhlsberger, ${ }^{3}$ Eckhart Förster, ${ }^{1,2}$ Thomas Stöhlker, ${ }^{1,2}$ Ingo Uschmann, ${ }^{1,2}$ and Gerhard G. Paulus ${ }^{1,2}$

1) Institut für Optik und Quantenelektronik, Friedrich-Schiller-Universität Jena, Max-Wien-Platz 1, D-077ł3 Jena, Germany

${ }^{2)}$ Helmholtz-Institut Jena, Fröbelstieg 3, D-07743 Jena, Germany

3) Deutsches Elektronen-Synchrotron DESY, Notkestraße 85, 22607 Hamburg, Germany

4) European Synchrotron Radiation Facility, CS 40220, 71, avenue des Martyrs, F-38043 Grenoble Cedex 9, France

(Dated: 5 October 2017; Received 24 May 2016; accepted 1 September 2016; published online 20 September 2016)

We report on the use of synthetic single-crystal diamonds for high purity x-ray polarimetry to improve the polarization purity of present-day x-ray polarimeters. The polarimeter setup consists of a polarizer and an analyzer, each based on two parallel diamond crystals used at a Bragg angle close to $45^{\circ}$. The experiment was performed using one (400) Bragg reflection on each diamond crystal and synchrotron undulator radiation at an x-ray energy of $9838.75 \mathrm{eV}$. A polarization purity of $8.9 \times 10^{-10}$ was measured at the European Synchrotron Radiation Facility (ESRF), which is the best value reported for two-reflection polarizer/analyzer setups. This result is encouraging and a first step to improve the resolution of x-ray polarimeters further by using diamond crystal polarizers and analyzers with four or six consecutive reflections.

The polarization rotation of light in a sucrose solution was one of the first applications of polarized light and is still one of the most famous experiments in optics, revealing the chirality of complex molecules. Today there are many applications based on polarization effects close to the visible regime of light, like ellipsometry in solid state physics, fluorescence polarization in chemistry or scanning laser polarimetry in medicine. In the x-ray regime, applications based on polarization effects are becoming increasingly significant and make use of the high degree of polarization provided by synchrotron or free-electron laser (FEL) sources. ${ }^{1-3}$ One prominent example is x-ray magnetic circular dichroism. ${ }^{4}$ More recently, the development of high-performance x-ray polarizers for crossed polarizer/analyzer setups expanded the applications of nuclear resonant scattering ${ }^{5}$ and even enabled the detection of optical activity of sugar at a photon energy of $12.9 \mathrm{keV} .{ }^{6}$ In order to detect such polarization effects in the x-ray range, appropriate instruments with high angular resolution near the extinction position are essential. Further improvements of x-ray polarizers will likely yield new physical insights and open up experiments like the detection of vacuum birefringence ${ }^{7-9}$ or the detection of huge magnetic fields $\left(\sim 10^{3} \ldots 10^{4}\right.$ Tesla $)$ in solid-density plasmas via Faraday rotation at $10 \mathrm{keV}$ x-ray energies.

In the first decades after the discovery of polarized x-rays by Charles Barkla in $1903,{ }^{10}$ not too many applications were found for $\mathrm{x}$-ray polarimetry. The brilliant $\mathrm{x}$-ray synchrotron sources gave rise to the development of $\mathrm{x}$ ray polarizers and analyzers by Hart et al. and Hrdý et al. in the seventies of the last century. ${ }^{11,12}$ Highly linear polarized radiation can be produced, if the Bragg

\footnotetext{
a) Electronic mail: hendrik.bernhardt@uni-jena.de
}

angle is close to the Brewster angle which is very close to $45^{\circ}$ for x-rays. In this case, the integrated reflectivity $\left(R_{\pi}\right)$ of the polarization state parallel to the diffraction plane ( $\pi$-polarization state) equals zero whereas the integrated reflectivity $\left(R_{\sigma}\right)$ of the polarization state perpendicular to the diffraction plane ( $\sigma$-polarization state) remains nearly unchanged, resulting in a suppression of the $\pi$-polarization. This is described by dynamical theory of diffraction, from which we can derive the relation $R_{\pi} \backsim \cos (2 \theta)$. The intensity ratio $I_{\pi} / I_{\sigma}$ of a x-ray beam is called polarization purity and is influenced by the ratio $R_{\pi} / R_{\sigma}$ of the used crystal polarizers. In addition, high reflectivity is achieved within the total reflection range of a single crystal rocking curve. To achieve maximum polarization purity, for any given family of lattice planes, the wavelength of the $\mathrm{x}$-rays has to be tuned until a Bragg angle of $45.00^{\circ}$ is reached.

The reachable polarization purity can be further improved by using several successive Bragg reflections between the walls of a groove cut in a perfect crystal, which is called channel-cut. This is analogous to the approach in optics, where light can be very well polarized by multiple transmission through a pile of glass plates, even though each plate is only a partial polarizer. ${ }^{13}$ By applying this method, Marx et al. measured polarization purities of $(2.4 \pm 0.9) \times 10^{-10}$ and $(5.7 \pm 2.1) \times 10^{-10}$ at photon energies of $6457 \mathrm{eV}$ and $12914 \mathrm{eV}{ }^{6}$ Those values were achieved with two silicon channel-cut crystals, each containing six consecutive (400) or (800) Bragg reflections, respectively. In order to detect very weak polarization effects, like a birefringence smaller than $10^{-12}$ induced in a quantum vacuum by high electromagnetic fields, ${ }^{9}$ an improvement of the polarization purity as compared to the existing results is essential. In the following, we present evidence that a higher polarization purity can be reached with perfect diamond crystals. 


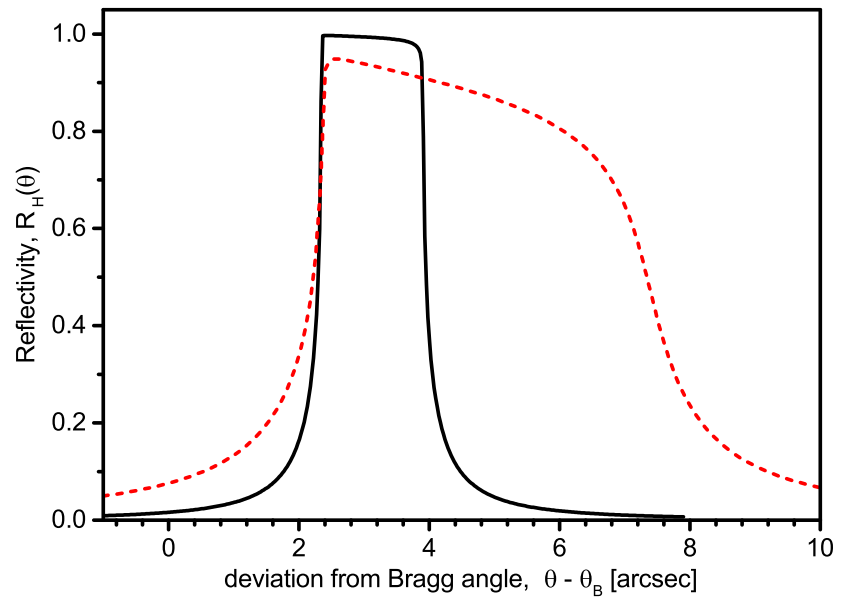

FIG. 1. Reflectivity of x-rays for the $\sigma$-polarization in $45^{\circ}$ symmetric Bragg scattering geometry as a function of the angle of incidence $\theta$, according to dynamical theory calculations. ${ }^{14}$ Solid line: the (400) Bragg reflection in diamond for $\mathrm{E}_{H}=9831 \mathrm{eV}$. Dashed line: the (400) Bragg reflection in silicon for $\mathrm{E}_{H}=6457 \mathrm{eV}$, as used by Marx et al.

Diamond offers a set of unique physical properties which are quite interesting for X-ray science, e.g. low $\mathrm{x}$-ray absorption, high thermal conductivity and high $\mathrm{x}$ ray reflectivity. ${ }^{15}$ Due to the production of artificial diamonds with reasonable good quality, diamond has become very attractive for use as monochromator crystals in high power density beamlines ${ }^{16,17}$, as material for phase plates ${ }^{18,19}$, beam splitter ${ }^{20}$ and as self-seeding crystals in FEL technology. ${ }^{21}$

In addition, diamond has properties that enable a further improvement of the polarization purity and eventually the resolution of the polarimeter setup. The reachable polarization purity is limited due to multiple-beam cases, where more than two reciprocal lattice points lie in the vicinity of the Ewald sphere. ${ }^{5}$ As a consequence, the incident beam excites a secondary reflection in addition to the desired primary one. If this secondary reflection is diffracted in the direction of the desired primary beam path (via Umweganregung) a degradation of the polarization purity may result.

The influence of multiple-beam cases can be reduced by an optimization of the crystal azimuth. To this end, the diffracting crystal is rotated around the reciprocal lattice vector to a region where possible secondary reflections are sufficiently far away. However, there is still a weak contribution from the tails of multiple reflections. The intensity of these contributions is proportional to $Z^{4}$, where $Z$ is the atomic number. ${ }^{22}$ That means the intensity of multiple reflections could be up to 30 times smaller in diamond as compared to silicon.

Another advantage of diamond is its high Bragg reflectivity which was remarkable demonstrated by Shvydkoet al.. For comparison Figure 1 shows the reflectivity curves of diamond (solid line) and silicon (dashed line) of the (400) Bragg reflection for $\theta_{B}=45^{\circ}$ according to the dynamical theory of diffraction. Due to the smaller lattice parameter and smaller structure factor of diamond as well as the resulting higher photon energy, the integrated reflectivity of diamond is much smaller as compared to silicon. On the other hand the peak reflectivity of diamond is nearly $100 \%$ for perfect crystals, which is important for a channel-cut crystal where the intensity is reflected several times.

All these advantages come with a number of severe drawbacks, as poor crystal quality and difficulty to get, especially large diamonds. In the past, high-temperature high-pressure diamonds were synthesized, providing high crystal quality and good applicability in x-ray optics as mentioned above. ${ }^{23}$ These diamonds are very expensive and rare due to individual production and the complicated growth procedure. In recent years, it was reported that diamond crystals of large size and good quality could be synthesized by chemical vapour deposition (CVD) with reasonable effort. ${ }^{24-27}$ Single crystal diamonds with sizes up to $8 \times 8 \times 1.2 \mathrm{~mm}^{3}$ are commercially available.

The real structure of CVD diamond crystals used in the present experiment was examined by visible light polarization analysis and x-ray topography. Both methods provide similar space-resolved information on crystal imperfections induced by strain which could reduce the quality of x-ray diffracted waves and degrade the polarization state of the radiation. Topographs show that the crystals used provide a relatively large central area of about $6 \times 6 \mathrm{~mm}^{2}$ where the Bragg reflection is homogeneous. Diffraction contrasts at the crystal borders show that strain is strongly increased at the outer crystal volume which should therefore not be used for Bragg reflection. Rocking curve measurements show that the measured rocking curve is broadened by 0.4 arcsec as compared to the theoretical predictions for a perfect crystal. This corresponds to a broadening of the diffracted energy width of $\Delta E / E=2 \times 10^{-6}$ and thus will not decrease the polarization purity. ${ }^{28}$

In the absence of large and perfect diamond crystals, it is not possible to manufacture a monolithic diamond channel-cut crystal. Thus, we used a special technique to realize the channel-cut geometry with two separate crystals. The scheme is known as a quasi channel-cut (QCC) and similar to the tuneable x-ray polarizer reported in Ref. 29. The QCC holder (Fig. 2 (a)) consists of two arms (2\& 3) with one CVD diamond crystal (1) mounted on each arm. The first crystal is fixed such that only an azimuthal rotation is possible (4) whereas the second crystal is attached to a mirror mount (5) allowing to tilt it such that it is parallel to the first crystal. With a feedback system consisting of a piezo element (6) and a capacitive sensor (7), the two arms of the QCC holder can be tilted in the diffraction plane like the two arms of a clothespin with subarcseconds precision. Thermal drifts tilting the crystals with respect to each other can be compensated with this closed-loop system. In order to minimize thermal expansion, most parts of the holder are made of invar steel. 

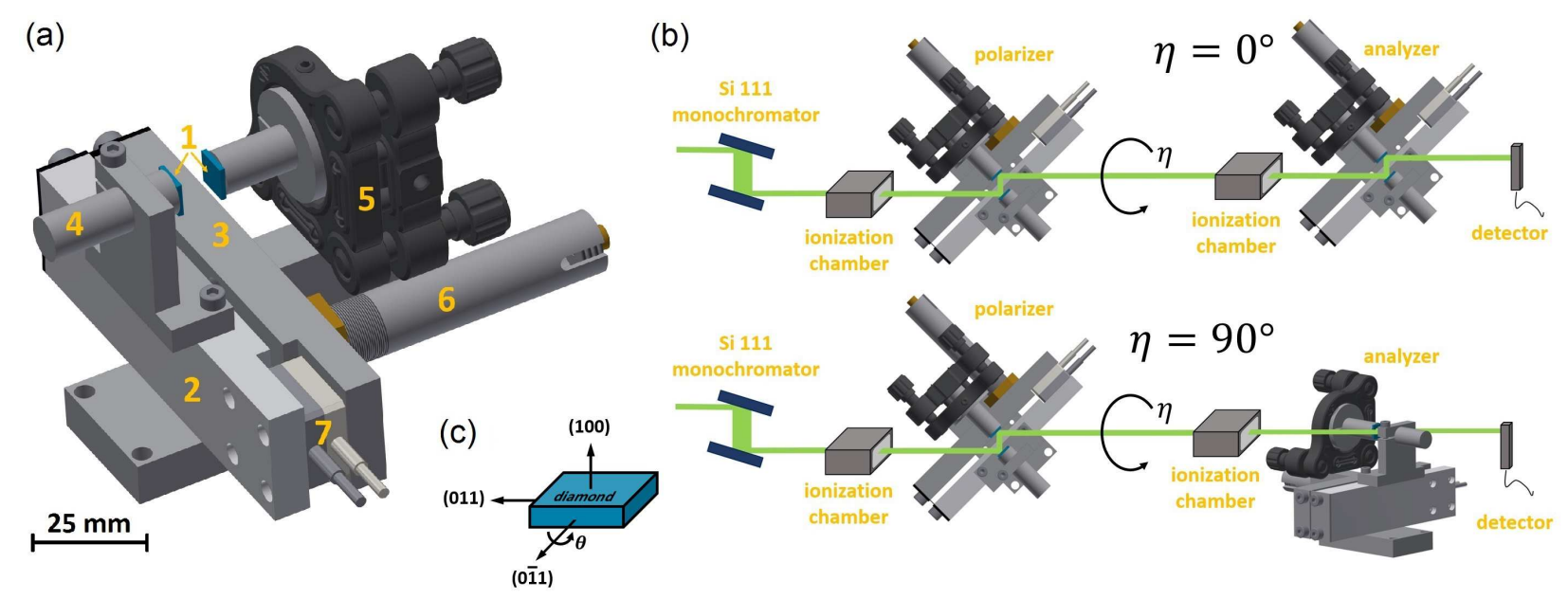

FIG. 2. (a) Quasi channel-cut holder for two separate CVD diamond crystals (1) consisting of two arms (2 \& 3$)$, a fixed crystal support (4), mirror support (5), piezo element (6) and capacitive sensors (7). (b) Scheme of the experimental setup: X-ray polarimeter with analyzer in transmission (upper part) and extinction (lower part) position. The mirror support pre-aligns the diamond crystals such that their (400) Bragg planes are parallel. The Piezo element with capacitative sensor feedback was then used to fine-tune this alignment. The whole assembly can be inclined to the Bragg angle of $45.00^{\circ}$. The analyzer assembly can additionally be rotated about the beam axis. (c) Lattice plane and surface orientation of the diamond crystals.

The entire QCC holder is mounted on a goniometer with 2 -circle segments and $x$ - $y$-translation stages. These devices enable a precise adjustment of the Bragg angle as well as the tilt and azimuth of the QCC with respect to the beam such that rocking curves can be recorded. One QCC serves as polarizer and the other as analyzer. The analyzer is equipped with an additional goniometer to rotate it around the incoming beam.

The full-step resolution of the goniometers and circle segments (tilt, azimuth) are 0.9 and 30 arcseconds, respectively. A scheme of the x-ray polarimeter setup is shown in Fig. 2 (b). For a detailed description see Ref. 28. Our measurement of polarization purity has been carried out at the Techniques and Instrumentation Test beam line (ID06) of the European Synchrotron Radiation Facility (ESRF) in Grenoble. The synchrotron was operating in the $7 / 8+1$ filling mode at a storage ring current of initially $200 \mathrm{~mA}$. The beam size was $\mathrm{x}_{\mathrm{ver}}=1 \mathrm{~mm}$ and $\mathrm{x}_{\text {hor }}=3 \mathrm{~mm}$. The undulator radiation was monochromatized by a double-crystal Si (111) high-heat load monochromator which leads to an energy bandwidth smaller than $1.5 \mathrm{eV}$. In order to reduce higher harmonics in the beam which could excite additional multiple-beam cases, the pitch of one of the monochromator crystals was slightly detuned relative to the other one. Before the main experiment, the energy of the high-heat load monochromator was calibrated at the $\mathrm{L}_{3}$ absorption edge of tantalum at $9881 \mathrm{eV}$. To select the optimum working energy, one of the diamonds was aligned with its edge (011-surface) in normal incidence to the x-ray beam (indicated in Fig. 2 (c)). The transmitted intensity of the (044) lattice plane was measured in Bragg geometry by rocking the crystal $0.5^{\circ}$ around the normal incidence position. Due to the Borrmann effect, Bragg reflections of different lattice planes were observed in the transmission scan. The scan was repeated for several photon energies of the undulator radiation until the transmitted intensity of all Bragg reflections was superposed in one peak and a Bragg angle of $90.00^{\circ}$ was reached for the (044) lattice plane. The resulting photon energy was $9835 \mathrm{eV}$. As a consequence of the symmetry of the diamond crystal structure, this is the same photon energy at which the (400) Bragg reflection occurs at an angle of $45.00^{\circ}$.

As described above, a pair of diamond QCC's has been used for the polarization purity measurement. Each quasi channel-cut contains two consecutive diamond (400) Bragg reflections. In order to optimize the extinction of the polarizer-analyzer setup, we slightly tuned the photon energy with the analyzer polarimeter in extinction position. We have found the highest extinction ratio at a photon energy of $9838.75 \mathrm{eV}$, i.e. $3.75 \mathrm{eV}$ above the value obtained by superposing all Bragg reflections, which we interpret as a uncertainty in the energy prealignment.

The polarization purity measurement, shown in Fig. 3 (a), was performed by rotating the analyzer QCC around the beam diffracted by the polarizer QCC. In each position, the rocking curve was measured by rocking the analyzer around the Bragg angle, while the polarizer remains at maximum Bragg reflectivity. The corresponding rocking curves normalized to the photon flux in front of the analyzer are plotted in Fig. 3 (b). At the position of crossed polarizer and analyzer $\left(\eta=90^{\circ}\right)$, a reflected intensity of 5 counts per second (cps) was measured at the maximum of the rocking curve with a background of $1 \mathrm{cps}$. The rocking curve maximum in parallel polarizer and analyzer position $\left(\eta=0^{\circ}\right)$ corresponds to $1.7 \times 10^{10} \mathrm{cps}$. In addition, it can be seen that the width of the rocking curves increases from 
(a)

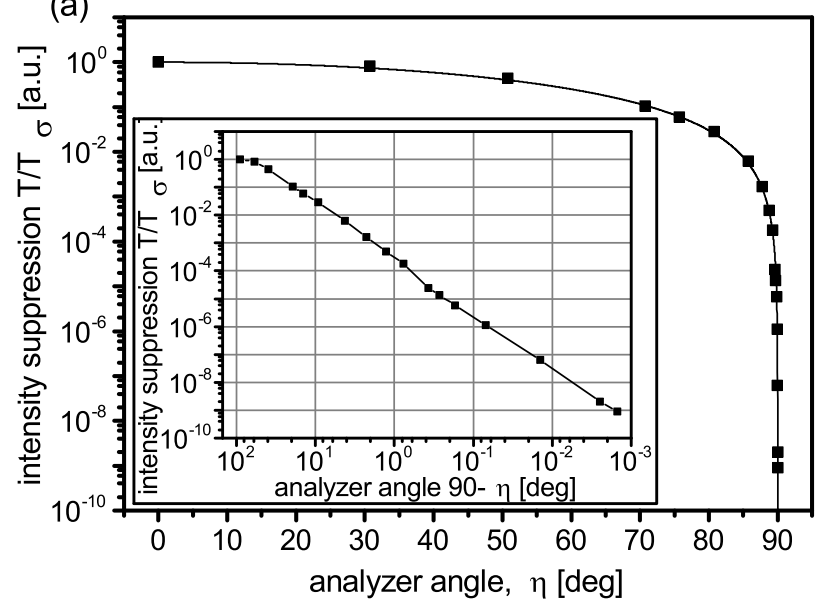

(b)

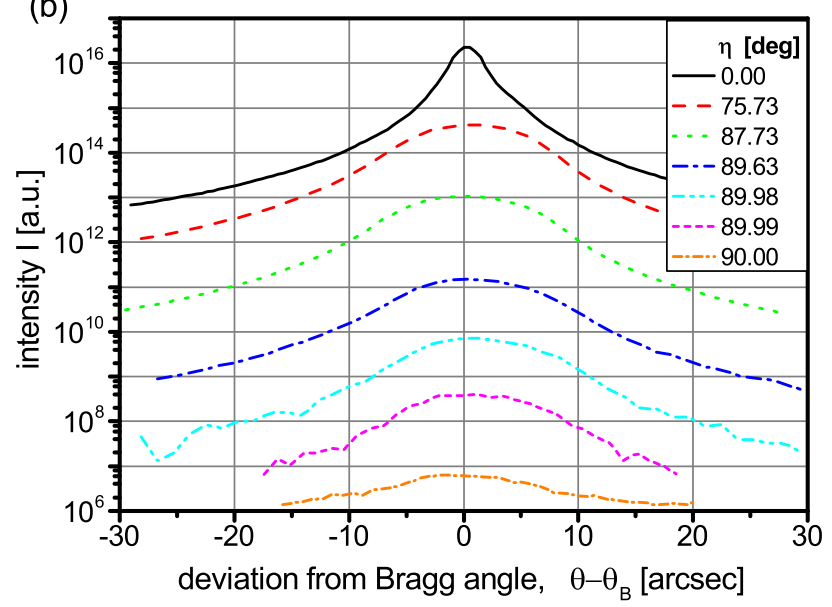

FIG. 3. (a) Measurement of the polarization purity of an x-ray beam with $9838.75 \mathrm{eV}$ photon energy using two-bounce diamond (400) Bragg reflection QCC's. Squares: integrated intensity of the analyzer rocking curve for different analyzer angles $\eta$ from $0^{\circ}$ (parallel position) to $90^{\circ}$ (crossed position) (b) Rocking curves of the diamond (400) QCC analyzer as a function of angle of incidence measured for different analyzer positions $\eta$. For each value of $\eta$, a rocking curve was measured and the reflected intensity was plotted versus the deviation from the Bragg angle. The plotted intensity was normalized to the incident intensity.

$2.4 \operatorname{arcsec}$ to $10 \operatorname{arcsec}$ as the angle $\eta$ increases from $0^{\circ}$ to $90^{\circ}$. This can be explained by the fact that the analyzer is leaving the dispersion plane of the polarizer when rotating around the $\eta$ axis. ${ }^{30}$ With polarizer and analyzer in crossed position, the rocking curve will be spectrally and angularly broadened due to the horizontal divergence of the photon beam.

Each data point in Fig. 3 (a) shows the integrated intensity of the rocking curve in the particular analyzer position $\eta$ normalized to the integrated intensity of the rocking curve in the parallel direction (analyzer position $\eta=0^{\circ}$ ). In order to cope with the vastly different photon count rates (ten orders of magnitude) as a function of $\eta$, two different detectors were used. For $0^{\circ} \leq \eta \leq 87.77^{\circ}$ a photodiode and for $87.77^{\circ} \leq \eta \leq 90.00^{\circ}$ an avalanche photodiode was used. We have achieved a polarization purity of $(8.9 \pm 1.8) \times 10^{-10}$.

The error is estimated to be $20 \%$ considering the error-prone contributions such as inhomogeneities of the detectors used, the matching of the photodiode and avalanche photodiode at $\eta=87.77^{\circ}$, drifts in the Bragg position of the crystals - which was minimized by checking the reflectivity of each crystal before each rocking curve measurement - and the accuracy of the energy adjustment.

The measured polarization purity of $8.9 \times 10^{-10}$ is the best value reported for two-reflection polarizer/analyzer setups, which justifies the approach to improve the polarization purity by using diamond crystals instead of silicon or germanium. In the field of high purity x-ray polarimetry, properties of the source like beam divergence, the amount of higher harmonics and the energy bandwidth of the used radiation as well as Umweganregungen and the quality of the used crystals play an important role. As we mentioned at the begin-
TABLE I. Best estimates by using physical limits for high purity x-ray polarimetry, two bounces, and the diamond (400) Bragg reflection.

\begin{tabular}{l|cc}
\hline \hline & $\begin{array}{c}3^{\text {rd }} \text { generation } \\
\text { synchrotron source }\end{array}$ & $\begin{array}{c}4^{\text {rd }} \text { generation } \\
\text { synchrotron source }\end{array}$ \\
\hline source & $\begin{array}{c}5 \times 10^{-10} \\
\text { divergence }\end{array}$ & $\begin{array}{c}2 \times 10^{-11} \\
\text { band width }\end{array}$ \\
\hline crystal & $\begin{array}{c}3 \times 10^{-11} \\
\text { imperfection }\end{array}$ & $\begin{array}{c}8 \times 10^{-12} \\
\text { multiple diffraction }\end{array}$ \\
\hline \hline
\end{tabular}

ning, the integrated reflectivity for the $\pi$-polarization state $R_{\pi}$ is proportional to $\cos (2 \theta)$ or $\cos ^{N}(2 \theta)$ for a channel-cut crystal with $N$ reflections, respectively. The beam divergence, energy bandwidth and the rocking curve broadening due to crystal imperfection can be considered as a deviation from the ideal Bragg angle of $45.00^{\circ}$. By using these limits, one can estimate the polarization purity for the case of two consecutive Bragg reflections $(N=2)$. In addition, we can calculate a limit of the polarization purity due to Umweganregung from the first and third harmonic of the undulator by using an algorithm described by Stetsko and Chang et al.. ${ }^{31}$ The major limits are summarized in Table I.

At a $3^{\text {rd }}$ generation synchrotron source, the polarization purity is mainly limited by the source divergence, which was more than $10 \mu \mathrm{rad}$ in our experiment and has a stronger influence than crystal imperfections. With a divergence of $1 \mu \mathrm{rad}$ at a $4^{r d}$ generation synchrotron source and perfect crystals, the energy bandwidth, will limit the polarization purity to $2 \times 10^{-11}$. If the third harmonic of the undulator is suppressed at least by a 
factor of $4 \times 10^{-6}$, Umweganregungen will contribute $8 \times 10^{-12}$. The influence of the beam divergence will be strongly reduced when four or six Bragg reflections are used instead of only two.

In conclusion, we have realized and tested a quasi channel-cut polarimeter with two reflections on diamond crystals. A polarization purity of $(8.9 \pm 1.8) \times 10^{-10}$ was achieved, i.e. a value very close to the record value previously set in using silicon channel-cuts, however with six reflections. The use of diamonds' makes wavelengths available to precision x-ray polarimetry that could not be addressed so far and, in particular, holds promise to even higher polarization purities if more reflections are used.

The authors thank O. Wehrhan and H. Marschner for crystal characterisation and $\mathrm{T}$. Köhler for providing the engineering drawings of the quasi channel-cut holder. This work was supported by the Deutsche Forschungsgemeinschaft (SFB-TR18), the Bundesministerium für Bildung und Forschung (05K13SJ1), and the Carl-ZeissStiftung.

${ }^{1}$ D. P. Siddons, M. Hart, Y. Amemiya, and J. B. Hastings, Phys. Rev. Lett. 64, 1967 (1990).

${ }^{2}$ B. Marx, K. S. Schulze, I. Uschmann, T. Kämpfer, O. Wehrhan, H. C. Wille, K. Schlage, R. Röhlsberger, E. Weckert, E. Förster, T. Stöhlker, and G. G. Paulus, Applied Physics Letters 105, 024103 (2014).

${ }^{3}$ B. A. Palmer, G. R. Edwards-Gau, B. M. Kariuki, K. D. Harris, I. P. Dolbnya, and S. P. Collins, Science 344, 1013 (2014).

${ }^{4}$ G. van der Laan and A. I. Figueroa, Coordination Chemistry Reviews 277, 95 (2014).

${ }^{5}$ T. S. Toellner, E. E. Alp, W. Sturhahn, T. M. Mooney, X. Zhang, M. Ando, Y. Yoda, and S. Kikuta, Applied Physics Letters 67, 1993 (1995).

${ }^{6}$ B. Marx, K. S. Schulze, I. Uschmann, T. Kämpfer, R. Lötzsch, O. Wehrhan, W. Wagner, C. Detlefs, T. Roth, J. Härtwig, E. Förster, T. Stöhlker, and G. G. Paulus, Phys. Rev. Lett. 110, 254801 (2013).

${ }^{7}$ F. Karbstein, H. Gies, M. Reuter, and M. Zepf, Physical Review D 92, 071301 (2015).

${ }^{8}$ H. Gies and L. Roessler, Physical Review D 84, 065035 (2011).

${ }^{9}$ T. Heinzl, B. Liesfeld, K.-U. Amthor, H. Schwoerer, R. Sauerbrey, and A. Wipf, Optics Communications 267, 318 (2006).

${ }^{10}$ C. G. Barkla, Philosophical Magazine Series 6 5, 685 (1903), http://dx.doi.org/10.1080/14786440309462976.

${ }^{11}$ M. Hart, Philosophical Magazine Part B 38, 41 (1978), http://dx.doi.org/10.1080/13642817808245319.
12 J. Hrdý, E. Krouský, and O. Renner, physica status solidi (a) 53, 143 (1979).

${ }^{13}$ M. Hart and A. R. D. Rodrigues, Philosophical Magazine Part B 40, 149 (1979), http://dx.doi.org/10.1080/13642817908246366.

${ }^{14}$ O. Lugovskaya and S. Stepanov, Soviet physics. Crystallography 36, 478 (1991).

${ }^{15}$ Y. Shvyd'Ko, S. Stoupin, V. Blank, and S. Terentyev, Nature Photonics 5, 539 (2011).

${ }^{16}$ L. E. Berman, J. Hastings, D. P. Siddons, M. Koike, V. Stojanoff, and M. Hart, Nuclear Instruments and Methods in Physics Research Section A: Accelerators, Spectrometers, Detectors and Associated Equipment 329, 555 (1993).

${ }^{17}$ P. Fernandez, T. Graber, W.-K. Lee, D. Mills, C. Rogers, and L. Assoufid, Nuclear Instruments and Methods in Physics Research Section A: Accelerators, Spectrometers, Detectors and Associated Equipment 400, 476 (1997).

${ }^{18}$ C. Giles, C. Vettier, F. De Bergevin, C. Malgrange, G. Grübel, and F. Grossi, Review of scientific instruments 66, 1518 (1995).

${ }^{19}$ V. Scagnoli, C. Mazzoli, C. Detlefs, P. Bernard, A. Fondacaro, L. Paolasini, F. Fabrizi, and F. de Bergevin, Journal of Synchrotron Radiation 16, 778 (2009).

${ }^{20}$ D. Zhu, Y. Feng, S. Stoupin, S. A. Terentyev, H. T. Lemke, D. M. Fritz, M. Chollet, J. Glownia, R. Alonso-Mori, M. Sikorski, et al., Review of Scientific Instruments 85, 063106 (2014).

${ }^{21}$ E. Saldin, E. Schneidmiller, Y. V. Shvyd'ko, and M. Yurkov, Nuclear Instruments and Methods in Physics Research Section A: Accelerators, Spectrometers, Detectors and Associated Equipment 475, 357 (2001).

${ }^{22}$ J. Tischler and B. Batterman, Acta Crystallographica Section A: Foundations of Crystallography 42, 510 (1986).

${ }^{23}$ R. C. Burns, A. I. Chumakov, S. H. Connell, D. Dube, H. P. Godfried, J. O. Hansen, J. Härtwig, J. Hoszowska, F. Masiello, L. Mkhonza, M. Rebak, A. Rommevaux, R. Setshedi, and P. V. Vaerenbergh, Journal of Physics: Condensed Matter 21, 364224 (2009).

${ }^{24}$ F. Silva, J. Achard, O. Brinza, X. Bonnin, K. Hassouni, A. Anthonis, K. De Corte, and J. Barjon, Diamond and Related Materials 18, 683 (2009).

${ }^{25}$ Y. Mokuno, A. Chayahara, and H. Yamada, Diamond and Related Materials 17, 415 (2008).

${ }^{26}$ Y. Mokuno, A. Chayahara, H. Yamada, and N. Tsubouchi, Diamond and Related Materials 18, 1258 (2009).

${ }^{27}$ M. Schreck, J. Asmussen, S. Shikata, J.-C. Arnault, and N. Fujimori, MRS Bulletin 39, 504 (2014).

${ }^{28}$ B. Marx, I. Uschmann, S. Höfer, R. Lötzsch, O. Wehrhan, E. Förster, M. Kaluza, T. Stöhlker, H. Gies, C. Detlefs, et al., Optics Communications 284, 915 (2011).

${ }^{29}$ M. Hart, D. Siddons, Y. Amemiya, and V. Stojanoff, Review of scientific instruments 62, 2540 (1991).

${ }^{30}$ O. Brümmer, C. Eisenschmidt, and J. Nieber, Crystal Research and Technology 17, 509 (1982).

${ }^{31}$ Y. P. Stetsko and S.-L. Chang, Acta Crystallographica Section A: Foundations of Crystallography 53, 28 (1997). 